\title{
Is There Any Benefit of Drain Placement on Postoperative Complications in Patients Undergoing the Sistrunk Procedure?
}

\author{
Talha Ahmed Qureshi ${ }^{1} \quad$ Anwar Suhail $^{1} \quad$ Syed Sajjad Ali Zaidi ${ }^{1} \quad$ Wasif Siddiq $^{1}$ \\ ${ }^{1}$ Section of Otolaryngology Head-Neck Surgery, Aga Khan University \\ Hospital, Karachi, Pakistan \\ Address for correspondence Talha Ahmed Qureshi, MBBS, Section of \\ Otolaryngology Head-Neck Surgery, Aga Khan University, Stadium \\ Road, Karachi-74800, Pakistan (e-mail: talha.ahmed@aku.edu).
}

Int Arch Otorhinolaryngol 2015;19:331-335.

\begin{abstract}
Keywords

- otorhinolaryngologic surgical procedure

- thyroglossal cyst

- drainage

- postoperative complications

- surgical wound infection

Introduction Same-day, outpatient Sistrunk procedure is commonly performed to manage thyroglossal duct cyst anomalies and may lead to postoperative complications. Surgical drains are placed to prevent complications, but recent observations show no advantage and rather increased health care costs and patient discomfort.

Objective The study evaluated if drain placement in the Sistrunk procedure offers any benefit on postoperative complications.

Methods A retrospective analysis of patient records having undergone same-day, outpatient Sistrunk procedure from 2004 to 2014 was done. Of 58 (38 male and 20 female) patients included, 38 did not have drains placed and the remaining 20 had drains placed. Mean and median age of patients was 18.1 and 13.5 years, respectively. Postoperative complications of patients with drains versus those without drains were statistically analyzed.

Results Overall, about $10 \%$ of patients had hematoma/seroma (H-S), with $6.9 \%$ of patients needing aspiration for $\mathrm{H}-\mathrm{S} ; 3.4 \%$ had wound infections; and $1.7 \%$ had pus formation. No statistically significant differences in Sistrunk-related complications between patient groups (with drain or without drain) were seen using Fisher exact (two-sided) test: H-S $(p=0.08)$; need for aspiration $(p=0.29)$; wound infection $(p>0.05)$; and pus formation $(p=0.35)$. Chi-square test also did not show any significant difference in the groups in terms of number of follow-ups.

Conclusion Surgical placement of a drain in the Sistrunk procedure does not seem to offer any advantage in terms of reducing common postoperative complications. Sameday Sistrunk procedure without any drain placement may be a safer alternative without necessitating hospitalization. More studies with larger sample size are needed for further substantiation.
\end{abstract}

\section{Introduction}

Thyroglossal duct cyst (TDC) is a common abnormality of the neck region and can occur at any age, though it is much more common in the pediatric population. ${ }^{1}$ The most definitive, efficacious management is achieved through the Sistrunk

received

January 28, 2015

accepted

February 23, 2015

published online

March 27, 2015
DOI http://dx.doi.org/ 10.1055/s-0035-1549156. ISSN 1809-9777. procedure, a widely accepted and choice surgical technique, which effectively removes the cystic lesion and reduces recurrence rates. ${ }^{2-5}$ Common postoperative complications associated with the technique include wound-related infection, pus/abscess, and hematoma/seroma (H-S) formation, with subsequent airway compromise. ${ }^{3,6}$ Surgical drains are
Copyright $(2015$ by Thieme Publicações License terms Ltda, Rio de Janeiro, Brazil 
placed in patients undergoing the Sistrunk procedure to prevent these postoperative complications, but hardly any studies have overtly assessed if drain placement is actually necessary. A recent case series in a pediatric population suggests that routine drain placement may not be necessary. ${ }^{7}$ As drain placement in the Sistrunk procedure may often necessitate postoperative hospitalization, leading to increased cost and patient discomfort, it is important to understand if drain placement offers any substantial advantage.

The rationale of our study was to assess if drain placement in the Sistrunk procedure makes any significant difference in the prognosis of postoperative complications. The issue assumes significance as a focus of head and neck surgeries (including the Sistrunk procedure) now is shifting toward outpatient, same-day, and ambulatory surgeries. $^{5,8,9}$ Studies demonstrate same-day surgery to be a safer, less costly, and reasonable alternative to admission surgery, without increased patient risk. ${ }^{8,9}$ With a general tendency to reduce hospital stays and with the Sistrunk technique being increasingly performed in same-day, outpatient settings, evaluating the impact of drain placement with the technique on postoperative complications and patient morbidity is essential. ${ }^{5,7}$

With this objective, we conducted a retrospective study at our hospital to explore if same-day, outpatient Sistrunk procedure performed without drain placement was a safe alternative versus the same procedure with drain placement. The study focused on postsurgical complications of the Sistrunk procedure in both groups (those with or without drain placement) and evaluated whether surgical drain offered any substantial benefit in the Sistrunk procedure, and if it was necessary.

\section{Methods}

Records of all patients who underwent the Sistrunk procedure for TDC surgery in the preceding 10 years (2004 to 2014) were retrieved, and data were searched and extracted. In total, 80 patient records of the Sistrunk procedure could be retrieved and were reviewed. Certain patients were excluded to standardize the patient population and avoid any bias in the study. All patients who had a bleeding disorder were excluded. All those who had active infection and pus in cyst requiring incision and drainage of the cyst along with Sistrunk procedure were also excluded. Patients who had incomplete surgery like removal of just the cyst without the tract including the body of the hyoid (which were documented as Sistrunk but were not actually Sistrunk) were also not included. Patients who in addition to Sistrunk procedure also underwent other surgical procedures were excluded (e.g., those who underwent papillary carcinoma surgery and proceeded to the Sistrunk procedure, or thyroidectomy/lymph node biopsies along with Sistrunk procedure, among others). Moreover, cases with missing data like no mention of placement or nonplacement of drain were also not included. Records missing follow-up information or clues regarding complications after the Sistrunk procedure were also not included.
Based on inclusion-exclusion criteria, of 80 records, only 58 patients were deemed fit for analysis, and the remaining 22 were excluded due to either missing data or inclusionexclusion criteria. Patients who were found suitable for analysis $(n=58)$ were separated into two groups: patients who did not have a drain placed $(n=38)$ during the Sistrunk procedure, and those who had a drain placed $(n=20)$. Of these 58 patients, 38 were males (65.5\%) and 20 (35.5\%) were females. The age of the patients varied from 1 to 53 years, with the mean and median age of patients being 18.1 and 13.5 years, respectively. Patient demographics are summarized in -Table 1.

All patients had same-day surgery under general anesthesia. The patients came in the morning, were operated upon, and were discharged by evening after their condition stabilized. Of the 58 patients, 13 (6 from the drain group and 7 from the no-drain group) were admitted for 1 night and discharged the next day but for reasons unrelated to complications, apparently based on patient or surgeon preference. Patient preference included those who had come from distant places and had late evening surgery, those who felt nauseous and had possibly not recovered well from anesthesia, and those who had anticipatory anxiety and reported feeling "uncomfortable" or "not feeling well." Patients who were admitted due to surgeon preference included one patient who had sickle cell anemia (who did not have a drain) for optimization and another patient (who had a drain) to observe for any anticipatory $\mathrm{H}-\mathrm{S}$.

Postoperatively, all patients were given antibiotics along with analgesics. Both groups of patients had homeostasis secured at time of surgery, and no major complication was noted. None of the patients developed any major complication like damage to great vessels, nerve damage, hypothyroidism, perforation of pharynx/esophagus, or injury to

Table 1 Patient demographics

\begin{tabular}{|l|l|l|}
\hline & Without drain & With drain \\
\hline Total no. of patients (\%) & $38(65.5 \%)$ & $20(34.5 \%)$ \\
\hline Males (\%) & $24(41.4 \%)$ & $14(24.1 \%)$ \\
\hline Females (\%) & $14(24.1 \%)$ & $6(10.3 \%)$ \\
\hline Age group distribution (y) & & \\
\hline$\leq 15(\%)$ & $23(39.7 \%)$ & $10(17.2 \%)$ \\
\hline $16-30(\%)$ & $8(13.8 \%)$ & $5(8.6 \%)$ \\
\hline$>30(\%)$ & $7(12.1 \%)$ & $5(8.6 \%)$ \\
\hline Age (y) & & \\
\hline Mean & $16.9(14.7)$ & $20.4(15.2)$ \\
\hline Median & 12.5 & 15.5 \\
\hline Minimum & 1 & 1 \\
\hline Maximum age & 53 & 49 \\
\hline $\begin{array}{l}\text { No. of patients } \\
\text { admitted overnight }\end{array}$ & $7(12.1 \%)$ & $6(10.3 \%)$ \\
\hline
\end{tabular}

Abbreviation: SD, standard deviation.

Note: All percent values are absolute percentages. Overall mean age (SD) was 18.1 (14.8) y and median age, $13.5 \mathrm{y}$. 
airway. None of the patients required any surgical exploration post-Sistrunk procedure, except minor wound exploration or incision and drainage of pus.

Both groups of patients were reviewed, and data were extracted for various postoperative Sistrunk-related complications, like presence of $\mathrm{H}-\mathrm{S}$ (and if the patients required aspiration for $\mathrm{H}-\mathrm{S}$ or were managed conservatively), wound infection, and pus formation. Data on number of follow-up visits by each patient was also extracted. Of the 58 patients, 3 did not return for follow-up and were assumed to be doing fine.

The extracted data were stored and analyzed using SPSS version 16 (Armonk, NY, IBM Corp). Pearson chi-square test was used to see the association of drain or no drain with gender, age, H-S, aspiration, wound infection, pus, and number of follow-up visits. The $t$ test was used to compare if there was any significant difference in mean age of patients in both groups. The parametric nature of the data was accessed statistically, and normality assumption was checked before application of $t$ test.

\section{Results}

Sistrunk procedure-related complications were minimal in both groups. Overall, about $10 \%$ of patients had $\mathrm{H}-\mathrm{S}, 6.9 \%$ of whom needed aspiration for $\mathrm{H}-\mathrm{S}$ and the rest were managed conservatively; $3.4 \%$ had wound infections; and $1.7 \%$ had pus collection that required wound exploration or incision and drainage. The comparative overall gross percentages of complications in both groups (drain versus no drain) did not show any significant difference ( $\mathrm{H}-\mathrm{S}, 0$ versus $15.8 \%$; aspiration needed, 0 versus $10.5 \%$; wound infection, 5.0 versus $2.6 \%$; pus formation, 5 versus $0 \%$; respectively). None of the patients who were admitted overnight (patient or surgeon preference) developed any of the complications discussed.

The chi-square test compared both group of patients in terms of any Sistrunk procedure-related complications (H-S, aspiration required, wound infection, pus, and number of follow-up visits). The groups did not show any statistical significant difference in Sistrunk-related complications in the Fisher exact (two-sided) test: H-S $(p=0.08)$, aspiration required ( $p=0.29$ ), wound infection ( $p>0.99$ ), and pus formation ( $p=0.35$; - Table 2 ). The chi-square (two-sided) test also did not show any statistically significant difference in number of follow-ups for both group of patients ( $p=0.81$; - Table 2$)$. No significant difference between groups could be found with respect to gender. The $t$ test compared mean age difference between patients with and without drain, and no significant difference in mean age could be seen ( $p=0.34$; - Table 2 ).

\section{Discussion}

Analysis of data suggests that there is no significant difference in complications post-Sistrunk procedure (infection, pus/ abscess, or $\mathrm{H}-\mathrm{S}$ ) between the drain and no-drain groups. Furthermore, no patient admitted overnight developed any of the complications discussed, which supports our methodology because the patients were admitted for reasons not related to complications and admissions were apparently due to patient and surgeon preferences. The findings of our study resonate with that of another recently published case series, which tried to ascertain if drain placement is at all necessary with the Sistrunk procedure for treating TDCs in the pediatric population. ${ }^{7}$ The case series evaluated 30 consecutive pediatric patients (mean age 7.4 years) who underwent the

Table 2 Comparison of Sistrunk procedure patient groups (with drain versus without drain)

\begin{tabular}{|c|c|c|c|}
\hline Aspect of comparison & No drain $(n=38)$ & With drain $(n=20)$ & $p$ Value (significance) \\
\hline \multicolumn{4}{|l|}{ Complications } \\
\hline H-S (\%) & $6(15.8 \%)$ & $0(0 \%)$ & $0.084(\mathrm{NS})^{\mathrm{a}}$ \\
\hline Aspiration need (\%) & $4(10.5 \%)$ & $0(0 \%)$ & $0.28(\mathrm{NS})^{\mathrm{a}}$ \\
\hline Wound infection (\%) & $1(2.6 \%)$ & $1(5 \%)$ & $>0.05(\mathrm{NS})^{\mathrm{a}}$ \\
\hline Pus (\%) & $0(0 \%)$ & $1(5 \%)$ & $0.345(\mathrm{NS})^{\mathrm{a}}$ \\
\hline \multicolumn{4}{|c|}{ Number of follow-up visits } \\
\hline One (\%) & $24(68.6 \%)$ & $13(72.2 \%)$ & \multirow[t]{3}{*}{$0.80(\mathrm{NS})^{\mathrm{b}}$} \\
\hline Two (\%) & $10(28.6 \%)$ & $4(22.2 \%)$ & \\
\hline Three (\%) & $1(2.9 \%)$ & $1(5.6 \%)$ & \\
\hline Mean age (SD) & $16.87(14.69)$ & $20.40(15.22)$ & $0.394(\mathrm{NS})^{\mathrm{c}}$ \\
\hline \multicolumn{4}{|l|}{ Sex } \\
\hline Male (\%) & $24(63.2 \%)$ & $14(70 \%)$ & \multirow[t]{2}{*}{$>0.05(\mathrm{NS})^{\mathrm{a}}$} \\
\hline Female (\%) & 12 (36.8\%) & $6(30 \%)$ & \\
\hline
\end{tabular}

Abbreviations: H-S, hematoma/seroma; NS, not significant; SD, standard deviation.

Note: All percent values are within group percentages. Overall mean age (SD) of participants was $18.09 \pm 14.84$.

${ }^{a}$ Fisher exact test

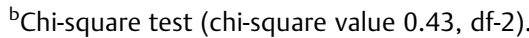

Independent samples $t$ text. 
Sistrunk procedure without drain placement (study group). Importantly, the first 10 patients were hospitalized, despite no drain placement, to observe for any complications in terms of hematomas, seromas, and subsequent airway compromise. However, when no major complications were seen, the next 20 patients undergoing the Sistrunk procedure without drain placement were treated with same-day, outpatient surgery. The study also had 21 age-matched controls (mean age 7.5 years) who underwent the Sistrunk procedure with drain placement. ${ }^{7}$ Interestingly, the study did not find any statistical difference in the complication rates between the study (no drain) and the control (drain) group (paired $t$ test, $p=0.85$ ). Conclusively, the author stated that surgical drain placement was not necessary in pediatric patients who underwent the Sistrunk procedure. ${ }^{7}$ Our study (which included both children and adults) reinforces the same finding in a pediatric population and extends the generalizability of similar outcome in the Sistrunk procedure performed in an adult population.

With hardly any studies exploring the need for drain placement in the Sistrunk procedure, no protocol recommendation or consensus seems to exist to place the drain or not. In fact, use of drains in thyroid/parathyroid surgery has been considered controversial and debatable and may be even contraindicated. ${ }^{10,11}$ It has been suggested that use of drains is not justified as drains cannot substitute for meticulous use of surgical technique and adequate hemostasis, in which noncompliance would eventually still lead to H-S. Rather, it has been contended that in noncomplicated surgeries with minimal drainage, placement of a drain could lead to a possible infection. ${ }^{12,13}$ Hence, nonusage of drains in the Sistrunk procedure is not only related to reduced hospital stay, less cost, and improved patient comfort, but also has implications in terms of decreased chance of postoperative infections.

Though not specific to the Sistrunk procedure, HurtadoLópez et al tried to analyze the actual value and effectiveness of a drain in thyroid surgery settings and found that presence or absence of drains did not affect the incidence of seroma or hematoma postsurgery. ${ }^{14}$ It is worth mentioning that although routine use of drains does not seem to be indicated in uncomplicated thyroid surgery cases, in complicated cases or when dead space is large, drains do have a use. ${ }^{15}$ The same may be applicable while using the Sistrunk procedure to surgically excise and manage TDCs, and the surgeon preference for drain placement should depend on merits of its use and not on routine use in the Sistrunk procedure.

Due to the increasing burden of extensive head and neck surgeries requiring mandatory hospitalization, smaller surgeries like the Sistrunk procedure be managed as same-day, outpatient treatment. As drain placement in the Sistrunk procedure may often require in-patient admission and increased hospital stay, it should not be performed if it does not offer any considerable advantage in decreasing postoperative complications. This would also have positive implications on reduced treatment expenditure and minimized patient distress.

Bratu and Laberge evaluated same-day TDC surgery (which included Sistrunk procedure) in 100 children in a retrospective review and found that outpatient surgery was a safe alternative to postoperative admission surgery in uncomplicated cases (no comorbidity, congenital defect, or bleeding disorders). ${ }^{5}$ Interestingly, drain placement was one of the factors that prolonged the length of hospital stay in patients. Another point worth emphasizing is that $38 \%$ patients had drains placed at surgeon discretion with no further details. ${ }^{5}$ Bratu and Laberge also stated that outpatient, same-day surgery was safe for routine TDC excision (including the Sistrunk procedure), but surgeons who were reluctant to use same-day surgery due to fear of complications needed a shift in behavior to increase outpatient TDC surgery. ${ }^{5}$ This further emphasizes the rationale of performing the Sistrunk procedure as same-day, outpatient surgery and emphasizes that the unnecessary routine use of drain placement with the Sistrunk procedure would necessitate admission surgery leading to waste of health resources.

The fact that very few complications were observed in our study even after discharge suggests that if homeostasis is adequately achieved, drain placement in Sistrunk procedure may not be necessary. However, to take care of any unprecedented postoperative hematoma and/or edema and subsequent airway compromise in patients undergoing same-day, outpatient Sistrunk procedure without any drain placement, the surgeon should take all precautions. All patients with TDC having same-day Sistrunk procedure should be asked to report any postoperative abnormality like swelling, purulent discharge, or fever postdischarge, as soon as possible and irrespective of the scheduled follow-up. This not only would ensure optimal utilization of health care resources to manage any adverse complication (if any arise) but also would provide cost-benefit information and improved comfort to the patient, ensuring greater patient satisfaction and better overall management of TDCs.

The observational nature is an important limitation of our study. Smaller sample size is another limitation, as complications of $\mathrm{H}-\mathrm{S}$ in the Sistrunk procedure are relatively rare. Due to the retrospective, observational study design, with no randomization possible, there may be a risk of selection bias. Although, to the best of our effort, we could not find any clinical or surgical variable to be associated with placement or nonplacement of a drain, its possibility cannot be completely ruled out. Furthermore, because all surgeries were performed by the consultant and residents under direct supervision of the consultant (and consultant as first assistant), there does not seem to be any performance bias. Apparently, drain placement with the Sistrunk procedure does not seems to be determined by the person performing the surgery or the surgeon's expertise, and rather appears to be governed by patient and surgeon preference. However, we cannot deny it in absolute terms.

A study with a larger sample population would further confirm the findings of our study. Ideally, however, a randomized trial comparing the Sistrunk procedure, with or without drain, should be performed to definitively understand if placing a drain is at all necessary in the Sistrunk procedure. This would help create a protocol recommendation and consensus among surgeons managing TDCs, with 
respect to deciding whether or not a drain should be placed during the Sistrunk procedure. To best of our knowledge, ours is the first study that has compared drain or no-drain Sistrunk procedure across all age groups; more comparative studies are needed in both pediatric as well as nonpediatric population.

\section{Conclusion}

Surgical placement of a drain did not seem to offer any advantage in patients undergoing the Sistrunk procedure at our hospital in terms of reduced postoperative complications. Hence, there does not seem to be any apparent need for drain placement when performing the Sistrunk procedure in patients with TDCs. Moreover, because no major complications were observed in surgically managing uncomplicated cases of TDCs by same-day, outpatient Sistrunk procedure without drain placement, it could be considered a safe alternative to the Sistrunk procedure with drain placement, which may require overnight hospitalization/admission. This would translate into better patient comfort, greater satisfaction, and reduced surgical costs in patients of TDCs undergoing the Sistrunk procedure.

\section{References}

1 Ward RF, Selfe RW, St Louis L, Bowling D. Computed tomography and the thyroglossal duct cyst. Otolaryngol Head Neck Surg 1986; 95(1):93-98
2 Sistrunk WE. The surgical treatment of cysts of the thyroglossal tract. Ann Surg 1920;71(2):121-122.2

3 Ghaneim A, Atkins P. The management of thyroglossal duct cysts. Int J Clin Pract 1997;51(8):512-513

4 Gallagher TQ Hartnick CJ. Thyroglossal duct cyst excision. Adv Otorhinolaryngol 2012;73:66-69

5 Bratu I, Laberge JM. Day surgery for thyroglossal duct cyst excision: a safe alternative. Pediatr Surg Int 2004;20(9):675-678

6 Maddalozzo J, Venkatesan TK, Gupta P. Complications associated with the Sistrunk procedure. Laryngoscope 2001;111(1):119-123

7 Hong P. Is drain placement necessary in pediatric patients who undergo the Sistrunk procedure? Am J Otolaryngol 2014;35(5): 628-630

8 Helmus C, Grin M, Westfall R. Same-day-stay head and neck surgery. Laryngoscope 1992;102(12 Pt 1):1331-1334

9 Laffaye HA. The impact of an ambulatory surgical service in a community hospital. Arch Surg 1989;124(5):601-603

10 Defechereux T, Hamoir E, Nguyen Dang D, Meurisse M. [Drainage in thyroid surgery. Is it always a must?] Ann Chir 1997;51(6): 647-652, discussion 653

11 Wihlborg O, Bergljung L, Mårtensson H. To drain or not to drain in thyroid surgery. A controlled clinical study. Arch Surg 1988; 123(1):40-41

12 Kristoffersson A, Sandzén B, Järhult J. Drainage in uncomplicated thyroid and parathyroid surgery. Br J Surg 1986;73(2):121-122

13 Ariyanayagam DC, Naraynsingh V, Busby D, Sieunarine K, Raju G, Jankey N. Thyroid surgery without drainage: 15 years of clinical experience. J R Coll Surg Edinb 1993;38(2):69-70

14 Hurtado-López LM, López-Romero S, Rizzo-Fuentes C, ZaldívarRamírez FR, Cervantes-Sánchez C. Selective use of drains in thyroid surgery. Head Neck 2001;23(3):189-193

15 Wax MK, Valiulis AP, Hurst MK. Drains in thyroid and parathyroid surgery. Are they necessary? Arch Otolaryngol Head Neck Surg 1995;121(9):981-983 